\title{
Bubble dynamics in turbulent duct flows: Lattice-Boltzmann simulations and drop tower experiments
}

\author{
Pau Bitlloch · Xavier Ruiz · Laureano Ramírez-Piscina · Jaume Casademunt
}

Received: date / Accepted: date

\begin{abstract}
Lattice-Boltzmann simulations of a turbulent duct ${ }_{19}$ flow have been carried out to obtain trajectories of passive tracers in the conditions of a series of microgravity experi- 20 ments of turbulent bubble suspensions. The statistics of these 21 passive tracers are compared to the corresponding measure- 22 ments for single-bubble and bubble-pair statistics obtained ${ }_{23}$ from particle tracking techniques after the high-speed cam- 24 era recordings from drop-towers experiments. In the condi- 25 tions of the present experiments, comparisons indicate that 26 experimental results on bubble velocity fluctuations are not 27 consistent with simulations of passive tracers, which points 28 in the direction of an active role of bubbles. The present 29 analysis illustrates the utility of a recently introduced exper- 30 imental setup to generate controlled turbulent bubble sus- 31 pensions in microgravity

Keywords turbulent flow $\cdot$ bubble dispersion - bubble interactions $\cdot$ microgravity $\cdot$ drop tower $\cdot$ lattice-boltzmann simulations
\end{abstract}

P. Bitlloch · J. Casademunt

Departament de Matèria Condensada,

Universitat de Barcelona,

Av. Diagonal 647, 08028-Barcelona, Spain

E-mail: pau.bitlloch@gmail.com

\section{Ruiz}

Departament de Química, Física i Inorgànica,

Universitat Rovira i Virgili,

Marcelí Domingo s/n, 43007-Tarragona, Spain

E-mail: josepxavier.ruiz@urv.cat

L. Ramírez-Piscina

Departament de Física,

Universitat Politècnica de Catalunya,

Doctor Marañón 44, 08028-Barcelona, Spain

E-mail: laure.piscina@upc.edu

X. Ruiz · L. Ramírez-Piscina · J. Casademunt $(\bowtie)$

Institut d'Estudis Espacials de Catalunya,

Gran Capità 2-4, 08034-Barcelona, Spain

E-mail: jaume.casademunt@ub.edu

\section{Introduction}

Multiphase flows are ubiquitous in technological applications. Specially complex situations correspond to the dispersion of one phase driven by a turbulent flow. In these cases the interaction between the flow and the dispersed phase is complicated by break-up and coalescence phenomena (Colin et al, 2008; Balachandar and Eaton, 2010). This problem is most relevant for space technologies such as life support systems and environmental control for life in space (Hurlbert et al, 2010), power generation and propulsion (Meyer et al, 2010) or thermal management (Hill et al, 2010). Therefore there is a strong interest in the study of turbulent bubbly flows under microgravity conditions (Colin, 2002).

Whereas there are several studies for the case of normal gravity (Kytömaa, 1987; Tryggvason et al, 2006; Mazzitelli et al, 2003), there are few works in microgravity (see for instance Colin et al (2001)). Very recently (Bitlloch et al, 2018) developed a gravity-insensitive method that generates monodisperse, homogeneous bubble suspensions in a turbulent duct flow. One important feature of this method regarding fundamental research in turbulent bubbly flows is the capability of controlling, in an independent way, important characteristics such as the degree of turbulence, the bubble size and also the bubble density.

In a series of microgravity experiments (36 drops of 4.7 s conducted in the ZARM Drop Tower), and by using particle tracking techniques, results on bubble velocity statistics were obtained (Bitlloch et al, 2018). One intriguing result obtained in these experiments was a weak dependence of the relative bubble velocity fluctuations on Reynolds number. Simple scaling arguments of developed turbulence do not predict such dependence. This anomalous scaling could then be either a property of the duct flow in the particular conditions of experiments, or be instead an indication of an active role of the bubbles on the flow. 
The main aim of this paper is to obtain precise numericalo2 results on turbulent duct flows in order to elucidate this ques-103 tion. To this end, Lattice-Boltzmann simulations of the flow 104 have been carried out. By using virtual passive tracers, these 105 simulations allowed to compare their statistics with that of $f_{106}$ the real bubbles. Simulation results also enabled to compare 07 the two-point statistics of passive tracers to that from the $e_{108}$ particle-tracking of bubble pairs. This gives interesting information on the flow mixing properties and the probability $y_{110}$ of bubble encounters. In particular we compared the char- ${ }_{111}$ acteristic times of separation between pairs of passive trac- ${ }_{112}$ ers in simulations and pairs of bubbles in the experiments ${ }_{113}$ All these information allowed to obtain an additional and $_{114}$ more accurate knowledge of the behavior of turbulent bub- $-_{115}$ bly flows under microgravity conditions.

\section{Lattice-Boltzmann simulations}

In order to characterize the structure and properties of a tur- ${ }^{120}$ bulent flow through a duct of square section we have per- ${ }^{121}$ formed 3D Lattice-Boltzmann simulations. The channel has ${ }^{122}$ been discretized into a uniform grid of 320x80x80 liquid ${ }^{123}$ nodes, representing a portion of $400 \times 100 \times 100 \mathrm{~mm}^{3}$ of the $\mathrm{m}^{124}$ duct, with periodic conditions at its ends. After some tests ${ }^{125}$ of various discretizations of the model, we decided for the ${ }^{126}$ D3Q15 Lattice Bhatnagar-Gross-Krook (BGK) model with ${ }^{127}$ mid-way wall boundary conditions for no-slip walls (Nour- ${ }^{128}$ galiev et al, 2003; Bitlloch, 2012).

For the sake of stability, since it is not possible to simu- ${ }^{130}$ late all scales of turbulence down to the Kolmogorov length, ${ }^{131}$ we used the Smagorinsky coefficient for sub-grid scale fil- ${ }^{132}$ tering (Hou et al, 1994). This method is based on the cal- ${ }^{133}$ culation of the local effective viscosity that would dissipate ${ }^{134}$ the sub-grid effects generated at each local point. Some rem- ${ }^{135}$ nant numerical instability was controlled by an additional ${ }^{136}$ smoothing procedure that preserved mass and momentum ${ }^{137}$ (Bitlloch, 2012).

The present code was parallelized and ran in the Mare $^{139}$ Nostrum supercomputer at the Barcelona Supercomputing ${ }^{140}$ Center (calculating typically with a set of 256 processors) ${ }^{141}$ and in a cluster of 16 processors at the Department of $A p{ }^{142}$ plied Physics of the Polytechnic University of Catalonia (UP( ${ }^{4}$ ) An overall estimation of the total CPU time used, account $-{ }^{144}$ ing for checking and optimization of the parallelized code $\mathrm{e}^{145}$ as well as for its subsequent simulations, has been of around $d^{146}$ 80,000 hours.

\subsection{Code checkings}

In order to check our code, we ran simulations for the same ${ }_{152}$ conditions as Pattison et al (2009). Comparisons showed 53 good agreement in the time-averaged structure of the flow,154 obtaining the same main longitudinal component of the flow and a reasonable agreement on the residual transversal components associated to the square section of the duct (secondary flow, see below), which was qualitatively correct except for small asymmetries probably due to insufficient temporal averaging.

As an illustrative example, Figure 1 shows the computed flow in a transversal section of the square duct for both Reynolds numbers of 3800 and 12700 . Lines represent the fluctuating component of the flow velocity $\left(\mathbf{u}^{\prime}=\mathbf{u}-\mathbf{U}\right)$. Length and color of the lines show the magnitude of each vector in an arbitrary scale. The same comparison is made for the longitudinal section of the flow placed at midway between walls in the $z$ direction is presented in Figure 2. Higher Reynolds numbers shows a finer and more detailed structure of turbulence that includes smaller eddies.

As measured by many authors (e.g., Melling and Whitelaw, 1976), and in contrast to the case of pipe flow with a circular section, turbulent flow in a square duct generates a weak remnant mean flow contained in the square transversal section of the flow, with pairs of symmetric vortices on each of the four edges of the channel. Those are called secondary flows, as they have a magnitude significantly smaller than the main longitudinal flow, and emerge only after careful time averaging of the transversal flow. Their structure is such that the flow approaches the edges from the bisector of the right angle between walls, then it follows the wall (moving really close to it) until it approaches the bisector of the wall, where it returns to the central part of the section. Figure 3 show the mean secondary flows obtained in our computations for the case of $\mathrm{Re}=3800$. Lines represent the flow vector $\left(0, U_{y}, U_{z}\right)$, being the length and color of the lines, the magnitude of the vector in an arbitrary scale. Results have been obtained from averaging over the whole length of the simulation, and over a period of 400,000 iterations (corresponding to $500 \mathrm{~s}$ of simulated time for the parameters of our experimental duct) after the simulation had reached the stationary regime. Given the difficulty of observing such secondary flows, they constitute a good test of the numerical simulation.

Analogously, we have done a statistical analysis for the computation with $\mathrm{Re}=12700$, averaging over a period of 300,000 iterations (corresponding, in our case, to $110 \mathrm{~s}$ of simulated time) after reaching the stationary solution of the flow. Comparing the numerical results obtained from both simulations in Figure 4 we find that the dimensionless profiles of velocity remain essentially unaltered by the change in the degree of turbulence in the flow. This is in agreement with the fact that the main structure of the flow is determined by the largest scales of turbulence, while the smaller ones define the scale of dissipation. The increase of the Reynolds number produces the decrease in size of the smallest scales of turbulence, resulting in the addition of more scales of ve- 

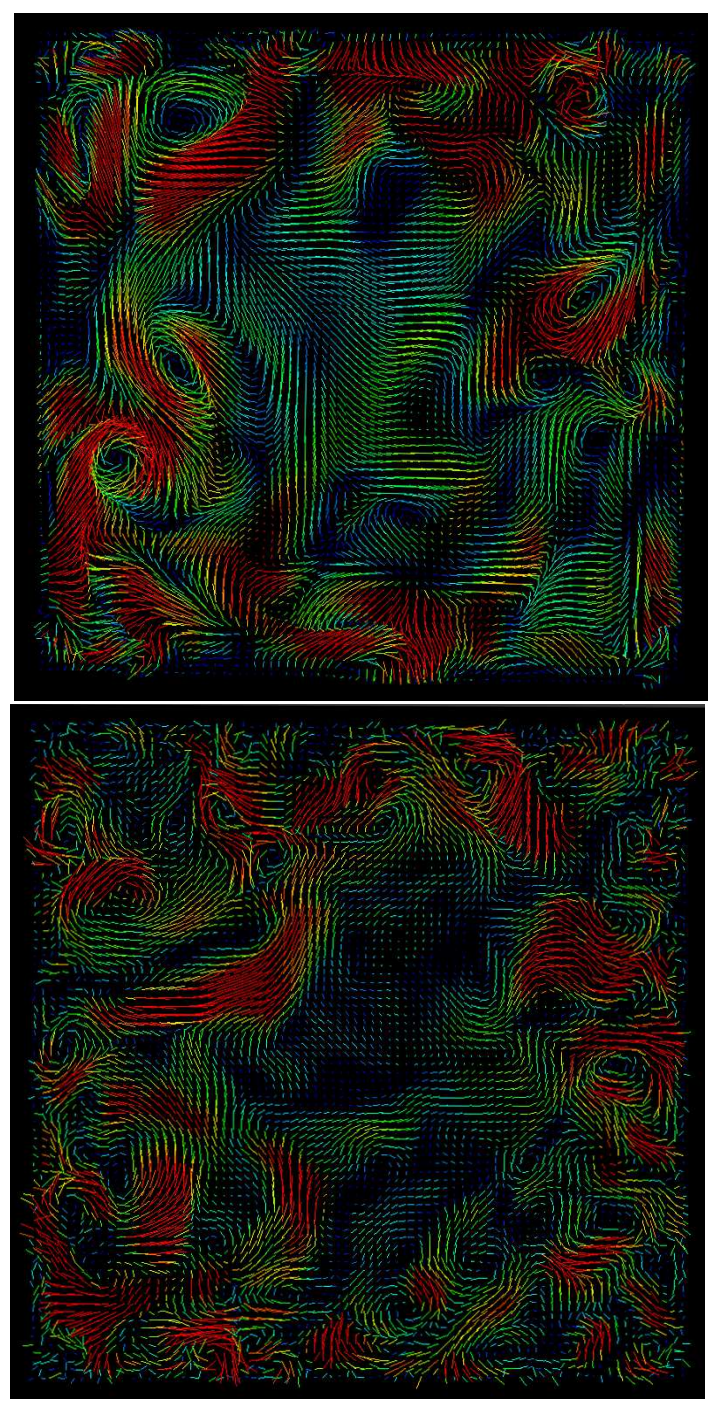

Fig. 1 Transversal sections of the turbulent flow. Lines and colors represent the direction and magnitude of the fluctuating component of the flow velocity $\mathbf{u}^{\prime}$ in arbitrary scale. (Above): $\mathrm{Re}=3800$. (Below): $\mathrm{Re}=12700$. Red indicates values $\geq 5$ times those of dark blue.

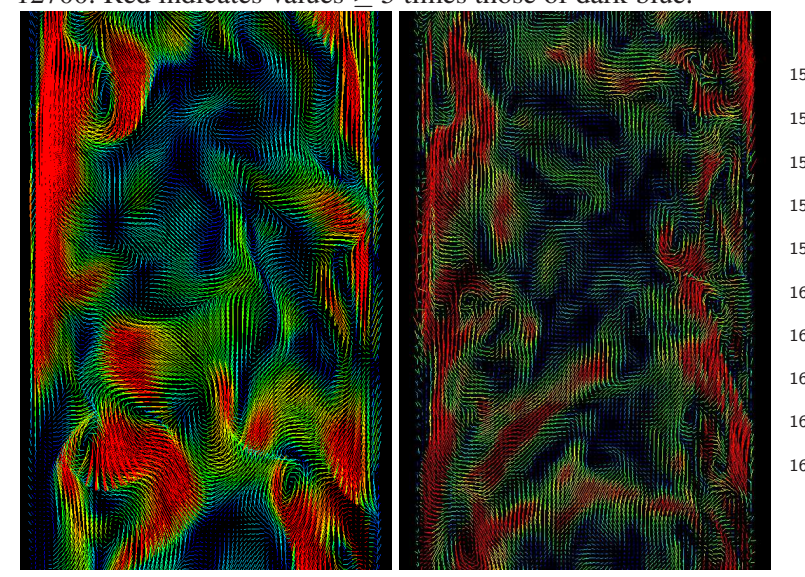

Fig. 2 Velocity fluctuations $\mathbf{u}^{\prime}$ on a longitudinal section of the duct $\mathrm{t}^{165}$ flow (xy plane)at $z=0.5 L_{c}$. Flow goes upwards. (Left): $\operatorname{Re}=3800$. (Right): $\mathrm{Re}=12700$.

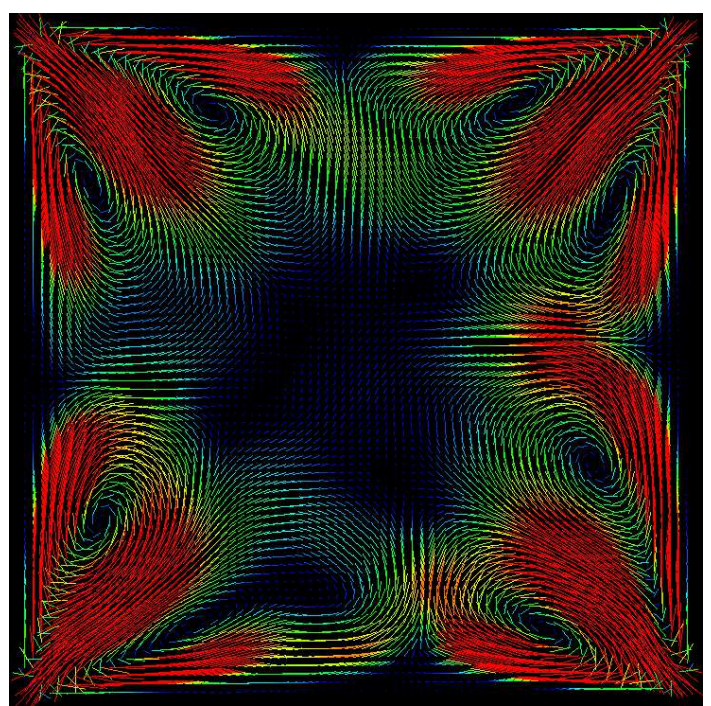

Fig. 3 Mean secondary flows on a transversal section of the duct (yz plane)for $\mathrm{Re}=3800$

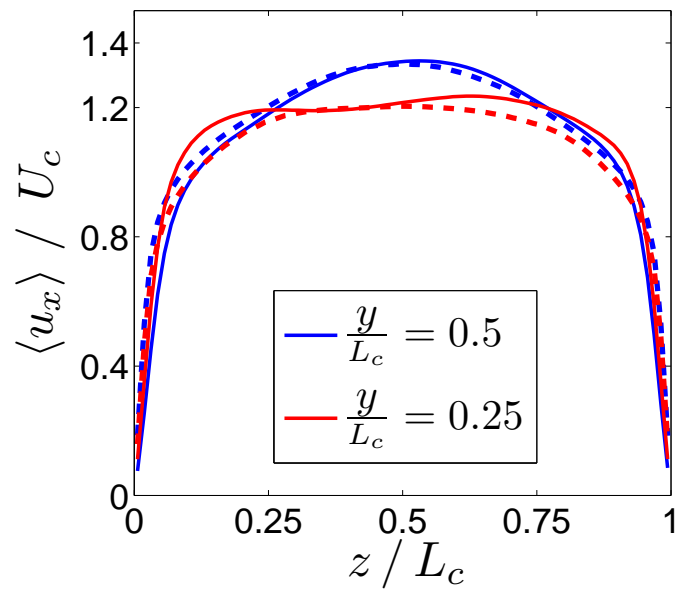

Fig. 4 Profiles of mean velocity component $\left\langle u_{x}\right\rangle$ at different sections $y / L_{c}$. Solid lines correspond to $\operatorname{Re}=3800$, dashed ones to $\operatorname{Re}=12700$

locity fluctuations that alter the fine, detailed properties of the flow, while the large scale structure remains unaffected.

Figure 5 shows the secondary components of the mean flow velocity for one of the simulations. It is easy to attribute the origin of the apparent asymmetries to the secondary flows of Figure 3, which would still require further statistical averaging to achieve convergence. Nevertheless, the figure is still interesting in order to realize the order of magnitude of the intensity of the secondary flows in relation to the main flow.

\section{Experimental details}

A complete description of the experimental device is presented in (Bitlloch et al, 2018), so only a short summary will be made here. The turbulent co-flow is generated by in- 


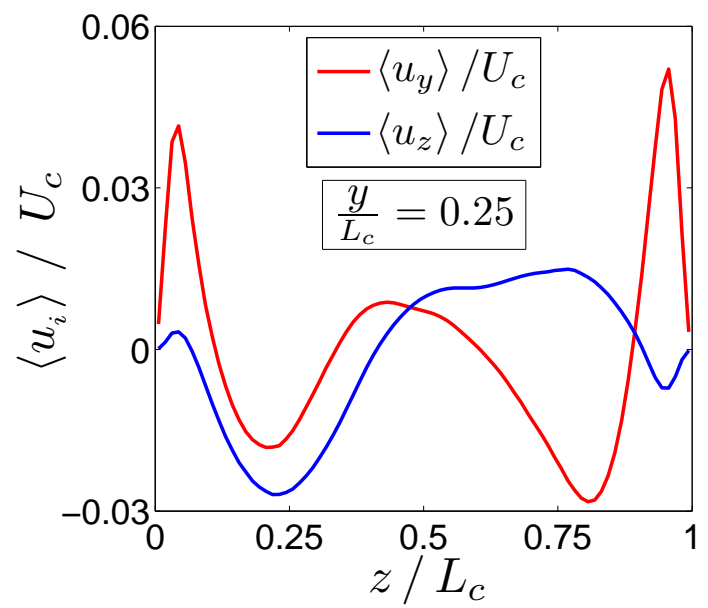

Fig. 5 Profiles of mean velocity components $\left\langle u_{y}\right\rangle$ and $\left\langle u_{z}\right\rangle$ for $\operatorname{Re}=$ 3800 at the section $y / L_{c}=0.25$

jecting water from nine inlets placed at the base of a vertical duct of square section and dimensions $800 \times 100 \times 100 \mathrm{~mm}^{3}$, and by using a wire mesh ( $2.5 \mathrm{~mm}$ thick) with square holes of $10 \times 10 \mathrm{~mm}^{2}$, corresponding to the scale of the most energetic eddies of the duct. The bubble suspension is achieved by injecting into the co-flow a pre-generated slug flow of water and air. This slug flow is formed by combining water and air flows in a T-junction device (Carrera et al, 2008), and is injected into the co-flow by four injectors forming a square. The bubble size is given by the size of the injectors, typically of the order of one millimeter, and can be finetuned through the injection parameters (Carrera et al, 2008; Arias et al, 2009; Bitlloch et al, 2015). The resulting Weber numbers are small enough for bubbles injected into the turbulent flow to be roughly spherical. Typical bubble sizes are larger than the dissipative turbulent scales, and therefore they could actively couple to the flow. At the same time bubbles are much smaller than the largest eddies, which are limited by the duct width of $100 \mathrm{~mm}$. Generated void fractions are typically small, of the order of a few percent. For the presented analysis, in order to reduce optical screening between bubbles, we have selected cases in the range from 0.3 to 0.8 void fractions.

This system is insensitive to the gravity level and permits ${ }^{206}$ to control the frequency and size of the generated bubbles in ${ }^{207}$ a way completely independent from the co-flow characteristics. More details on the setup can be found in Bitlloch et al (2018). An example of the injection of the bubbles can be seen in Fig. 6, with the resulting turbulent suspension shown $_{209}$ in Fig. 7

In order to analyze experimental results, images taken by high speed video cameras were processed by particle tracking techniques to reconstruct the bubble trajectories during the experiments. To this aim, after substracting the background, a standard filter was used to highlight the interphase of each bubble. In this way it was possible to identify the

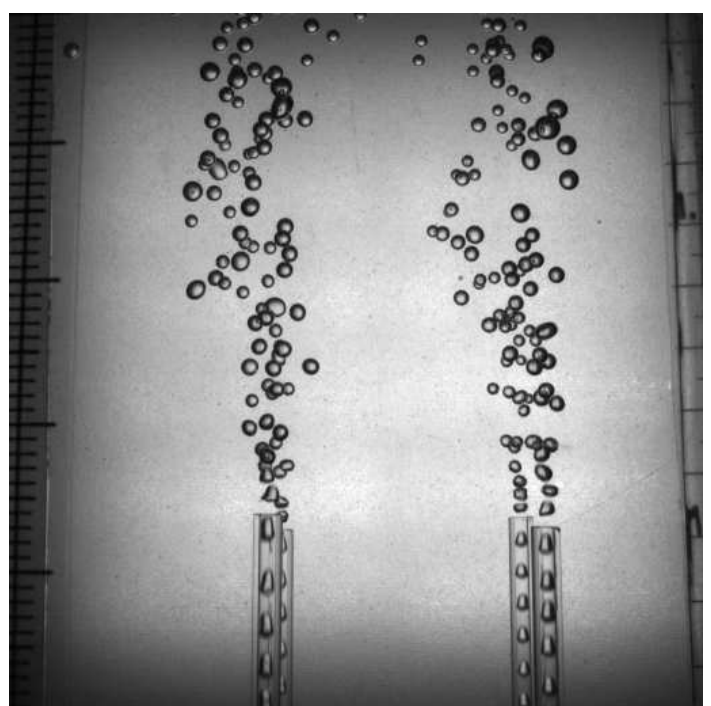

Fig. 6 Injection in microgravity by using flows of $Q_{l}=70 \frac{\mathrm{ml}}{\mathrm{min}}$ and $Q_{g}=46 \frac{\mathrm{ml}}{\min }\left(d_{B} \simeq 1.6 \mathrm{~mm}\right)$ in each injector, with a co-flow through the duct of $\operatorname{Re}=13000$.

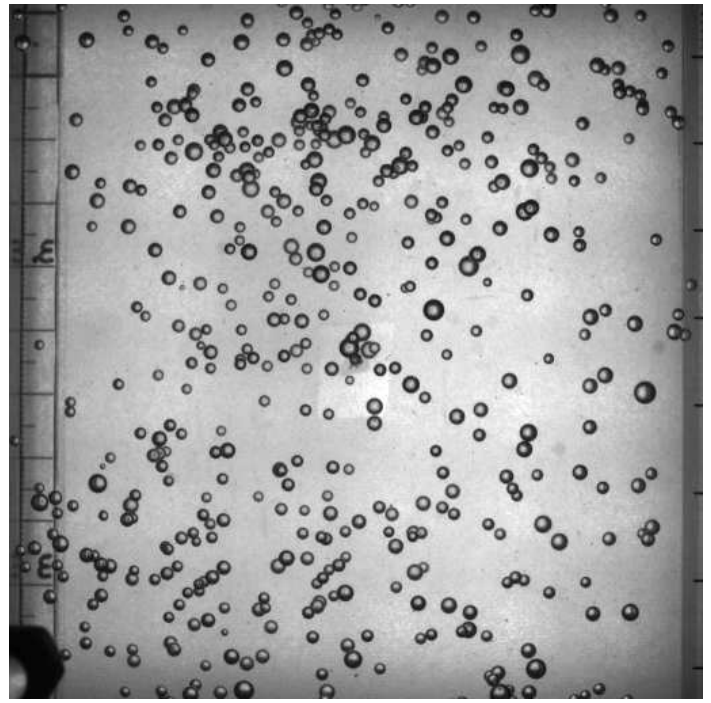

Fig. 7 Bubble suspension far from the injector achieved in microgravity in the same conditions as in Fig. 6.

trajectories of bubbles by tracking the white area strongly highlighted in their central part, which was surrounded and separated from the rest of bubbles by a clear interphase.

\section{Results}

4.1 Relative bubble velocity fluctuations

We have analyzed the fluctuations of each component of the relative bubble velocity. Specifically $\sigma_{i}$ is defined as the root-mean-square of the fluctuations of the $i$ component of the flow velocity:

$$
\sigma_{i}=\sqrt{\left\langle u_{i}^{\prime 2}\right\rangle}=\sqrt{\left\langle u_{i}^{2}\right\rangle-\left\langle u_{i}\right\rangle^{2}}
$$




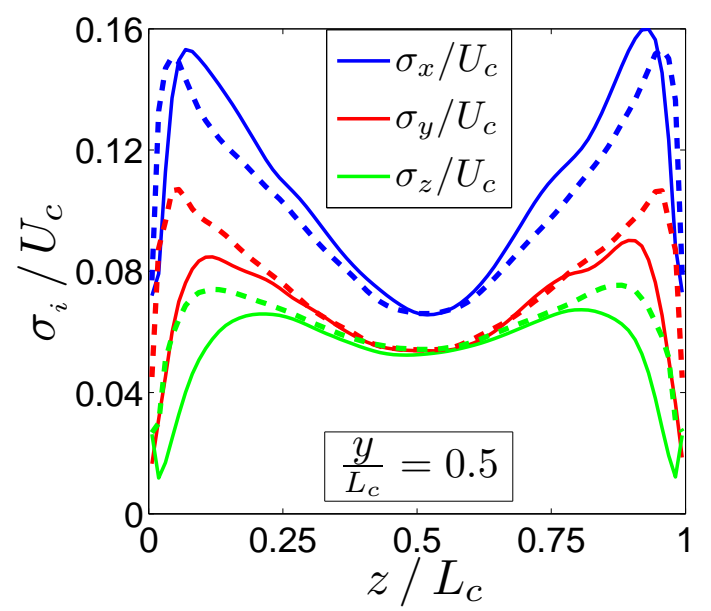

Fig. 8 Profiles of velocity fluctuations $\sigma_{i}$, at the section $y / L_{c}=0.5$. Solid lines correspond to $\operatorname{Re}=3800$, while dashed ones stand for $\operatorname{Re}=$ 12700

Previous experimental results, based on particle tracking ${ }_{242}$ techniques, concluded that the relative bubble velocity fluc $_{-243}$ tuations of the transversal $y$-component, $\sigma_{y}$, have a signifi- 244 cant decreasing tendency as the Reynolds number increases ${ }_{25}$ (Bitlloch et al, 2018). In particular, after relaxing the pseu- ${ }_{246}$ doturbulence generated by bubbles (due to their relative $\mathrm{ve}_{247}$ locity with respect the co-flow before switching-off gravity) $)_{248}$ it was found that the ratio $\sigma_{y} / U_{c}$ was 0.13 for $R e=6000_{249}$ whereas it was 0.08 for $R e=13000$ (Bitlloch et al, 2018) ${ }_{250}$ For the longitudinal component $\sigma_{x}$, however, the experimen ${ }_{251}$ tal data did not exhibit any conclusive tendency in this $\mathrm{re}_{-252}$ spect $\left(\sigma_{x} / U_{c}=0.10\right.$ for $R e=6000$ and $\sigma_{x} / U_{c}=0.11$ for $_{253}$ $R e=13000)$ (Bitlloch et al, 2018) .

To analyze these experimental results we study the pro- $_{255}$ files of both relative velocity fluctuation by using the present ${ }_{56}$ numerical results. Flow data in this case correspond to $\mathrm{re}_{257}$ sults that would exhibit passive tracers. Figures 8 and 9 show $_{258}$ these profiles taken at depths $\frac{y}{L_{c}}=0.5$ and 0.25 , respectively ${ }_{259}$ We then compare the relative fluctuations on each direction ${ }_{260}$ obtained in simulations for different degrees of turbulence. It ${ }_{261}$ can be seen that, in both cases, the change of the Reynolds ${ }_{262}$ number has no significant effect upon the relative velocity ${ }_{263}$ fluctuations. This is a result that coincides with the expec- ${ }_{264}$ tation from simple scaling arguments for fully developed ${ }_{265}$ turbulence, but that are not consistent with the mentioned ${ }_{266}$ experimental results. According to these results, bubbles $\mathrm{do}_{267}$ not seem to behave as passive traces of the flow, thus sug- ${ }_{268}$ gesting an active role of bubbles in the turbulence in the con $^{-669}$ ditions of the experiment.

\subsection{Behavior of pairs of bubbles}

In addition, to gain further insight into the dynamics of bub-275 ble suspensions in a turbulent flow, we studied the behaviour 76

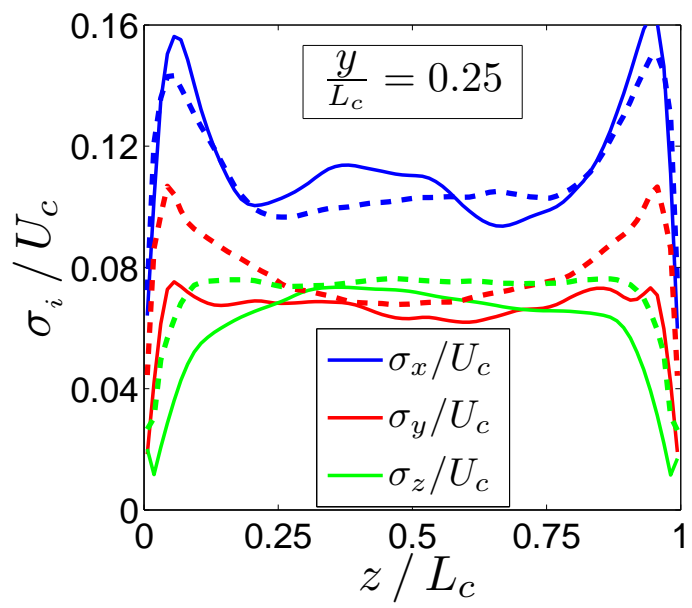

Fig. 9 Profiles of velocity fluctuations $\sigma_{i}$, at the section $y / L_{c}=0.25$. Solid lines correspond to $\operatorname{Re}=3800$ while dashed ones stand for $\operatorname{Re}=$ 12700

of pairs of bubbles and compared them to numerical predictions. To this aim we evolved by Lattice Boltzmann simulations an initially structured configuration of a large number of tracers (around 40000 tracers distributed in a regular lattice at relative distances of $1.25 \mathrm{~mm}$ ) for a long period of time, thus reaching a homogeneous distribution. Specifically, the case with $\mathrm{Re}=3800$ was first evolved during 30000 iterations (corresponding to $37 \mathrm{~s}$ of simulated time) and then the statistics was analyzed for the following 20000 iterations $(25 \mathrm{~s})$. The statistics of the case $\mathrm{Re}=12700$ was initiated after 50000 iterations (18.1 s), and spanned another 50000 iterations.

In Figure 10 we show a transversal coordinate as a function of time, and the projection on the transversal section of four trajectories described by tracers located initially on a close neighbourhood. The trajectories clearly show that the tracers remain close to each other for a certain finite time and then they strongly diverge from each other.

Experimental measurements of bubble pairs have been taken from the trajectories of bubbles previously captured with particle tracking methods. Those located at a distance smaller than $2 \mathrm{~mm}$ of another bubble (measured from their centers in the recorded image), have been considered a pair and have been used to calculate the averaged temporal evolution of their separation. In Figure 11 we display the evolution of the mean distance between pairs of bubbles at different temporal ranges of the microgravity experiments. Noisy signals at the final part of the lines denote a lack of sufficient statistics, caused by the high degree of screening between bubbles in the videos, which makes impossible to follow the trajectory of a bubble for a long period of time. Thus, as time increases, we are losing the track of more bubble pairs and consequently we get poorer statistics. In Figure 11top, for the smaller $R e=6000$, the slope of the mean separation versus time is steadily reducing in successive time 

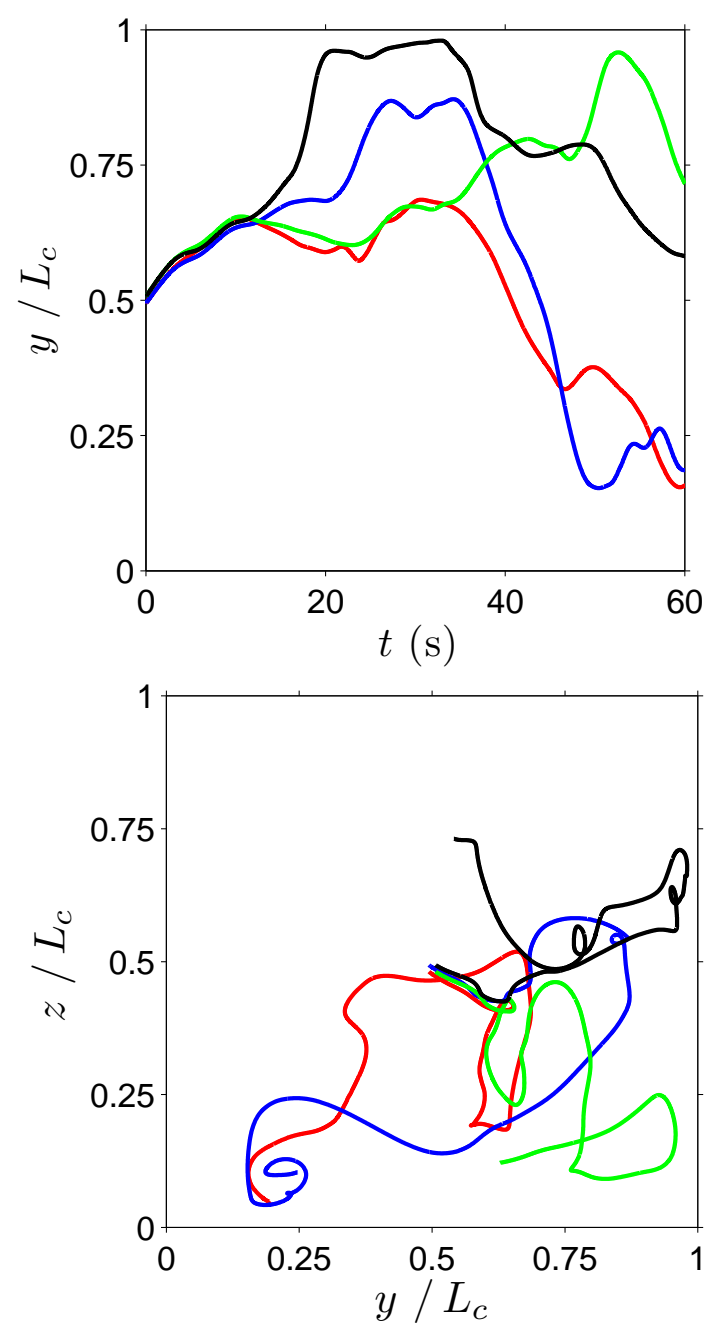

Fig. 10 Trajectories described by 4 passive tracers initially separated a distance of $1.25 \mathrm{~mm}$ of each other in a flow with $\mathrm{Re}=3800$ obtained from Lattice-Boltzmann simulations. (Top): transversal coordinate as a function of time; (Bottom): projection on the transversal section.

windows. It is important to recall that pseudo-turbulence is decaying during the experiment, as it was observed in Bitlloch et al (2018). The present results constitute another interesting manifestation of the same phenomena. In Figure 11bottom (larger co-flow velocity, with $R e=13000$ ) there are almost no differences in the short times for the first three time windows. In this case the intrinsic turbulence of the co-flow dominates so that the pseudoturbulence relaxation is more difficult to observe (which agrees with relaxation of velocity fluctuations being much weaker in this case as seen in Bitlloch et al (2018)). The different behavior observed in the last time window is due to the arrival of bubbles already generated in microgravity, and hence generated and transported in different conditions.

Figure 12 plots the mean separation of bubble pairs, measured after the first second of microgravity. Each line corresponds to a different set of injection parameters.
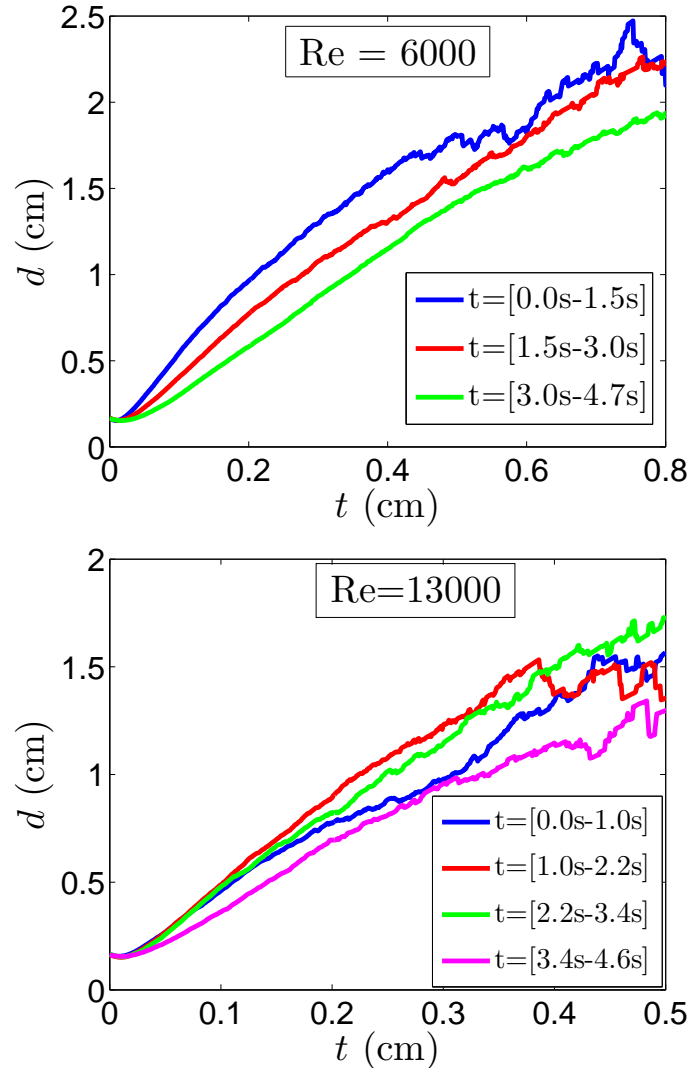

Fig. 11 Mean separation of pairs of bubbles. Each line correspond to a temporal range of the experiment in microgravity. (Top): Single experiment (D4) with $R e=6000$. (Bottom): Single experiment (D8) with $R e=13000$ (see Bitlloch et al (2018)).

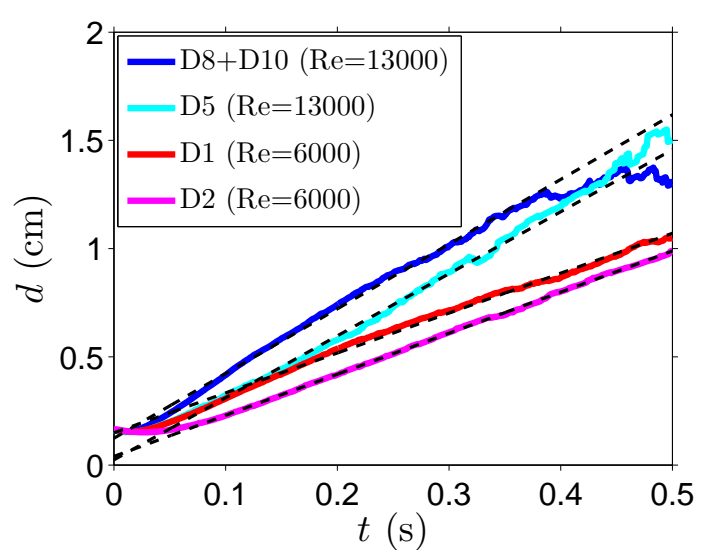

Fig. 12 Mean separation of pairs of bubbles for different parameters of injection. Solid lines correspond to measures from images taken by four video cameras at the indicated drops (see detailed parameters in Bitlloch et al (2018)). In the case of the dark blue line results from two equivalent experiments have been averaged. Dashed lines are fittings (described in Table 1) of the correspondent data. Results have been taken after the first second of microgravity. 
Namely dark and light red lines correspond to $\mathrm{Re}=6000$. and dark and light blue lines correspond to $\mathrm{Re}=13000$. We find that the measurements for equivalent degrees of turbulence share a similar slope once they have reached the linear regime, defining an effective rate of separation.

Dashed lines in Fig. 12 correspond to the linear fittings shown in Table 1. A clear dependence with Reynolds number can be observed on the rate of separation obtained in the fittings. For an increase of Re by a factor $\simeq 2.2$, the separation rate is increased by a factor $\simeq 1.6$.

\begin{tabular}{|c|c||c|c|}
\hline Param & $\operatorname{Re}$ & $d_{0}(\mathrm{~cm})$ & $v_{\text {sep }}(\mathrm{cm} / \mathrm{s})$ \\
\hline \hline$d_{x y}$ & 6000 & 0.04 & 1.90 \\
$d_{x y}$ & 6000 & 0.15 & 1.84 \\
\hline$d_{x y}$ & 13000 & 0.02 & 2.85 \\
$d_{x y}$ & 13000 & 0.12 & 2.99 \\
\hline
\end{tabular}

Table 1 Linear fittings of the form $d_{x y}=d_{0}+v_{\text {sep }} t$, for the mean separation in the plane xy between pairs of bubbles, used in Fig.12.

At this point it is important to call the attention upon the fact that the pairs of bubbles defined from experimental images are in many cases only apparent, due to the lack of information about the depth along the visual direction $z$. A majority of them are separated by distances much larger than the apparent separation and thus will follow rather independent trajectories. If we consider that a pair of bubbles is real when their initial separation $\Delta z_{0}$ in the visual direction is smaller than $1.6 \mathrm{~mm}$, for a homogeneous distribution of bubbles in our duct of width $L_{y}=100 \mathrm{~mm}$ we obtain a proportion of about $3 \%$ of real pairs, against $97 \%$ of apparent ones. One could think of different strategies to differentiate the two populations of pairs, with the help of a detailed statistical study of tracers in the simulations. However, due to the small statistical significance of the real pairs, the lack of more experiments to increase the amount of data makes any of such attempts virtually hopeless.

Figure 13 shows the evolution of the mean separation between real pairs of tracers, obtained from our simulations for two different degrees of turbulence. The first noticeable observation is that real pairs of tracers, unlike our experimen ${ }_{337}$ tal measures, have an average separation that grows closer $_{338}$ to exponentially in time. This rate is defined by an exponent 339 $\mathscr{L}$, which we may assimilate to an effective Lyapunov expo $_{340}$ nent, that controls the average rate of exponential separation ${ }_{34}$ $d(t)=d_{0} e^{\mathscr{L} t}$ of infinitesimally close trajectories in a chaotic ${ }_{422}$ dynamical system (Salazar and Collins, 2009). Fits in $\mathrm{Fig}_{343}$ ure 13 are shown in Table 2, which adjust nicely to simula 344 tions until the finite size effects of the duct section become ${ }_{345}$ important and slow down the growth, as can be observed $\mathrm{in}_{346}$ the figure for the most turbulent case.

In order to compare the experimental measurements with (h⿻ $_{48}$ those of simulations taken in equivalent conditions, we have 49

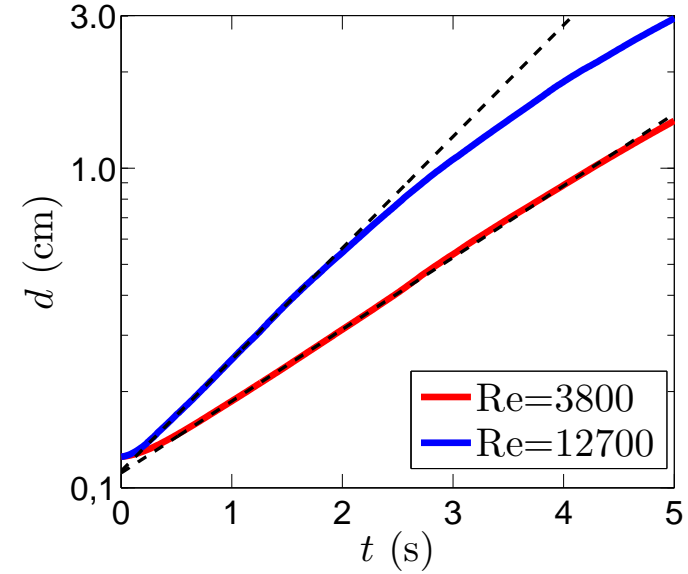

Fig. 13 Mean separation between real pairs of tracers. Distances on logarithmic scale. Fittings in dashed lines described in Table 2.

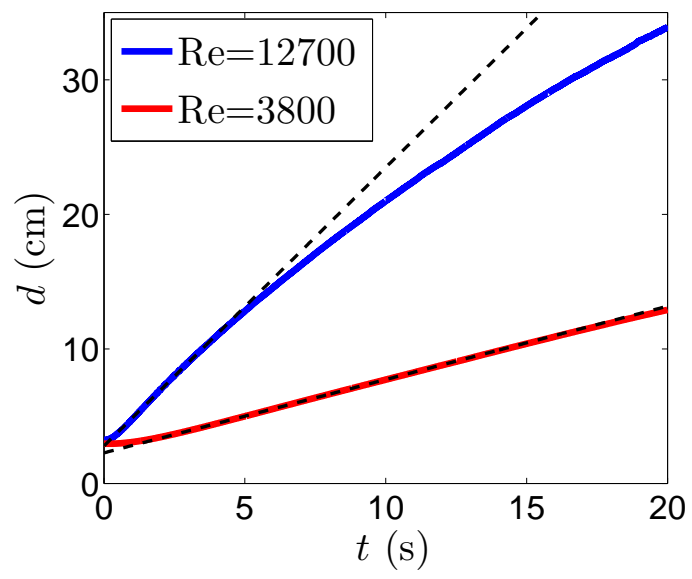

Fig. 14 Mean separation between apparent pairs of tracers (i.e., initial $\left.\Delta z_{0}>1.6 \mathrm{~mm}\right)$. Fittings in dashed lines described in Table 3 .

\begin{tabular}{|c|c||c|c|}
\hline Param & $\operatorname{Re}$ & $d_{0}(\mathrm{~cm})$ & $\mathscr{L}\left(\mathrm{s}^{-1}\right)$ \\
\hline \hline$d$ & 3800 & 0.11 & 0.52 \\
\hline$d$ & 12700 & 0.11 & 0.80 \\
\hline
\end{tabular}

Table 2 Exponential fittings of the form $d(t)=d_{0} e^{\mathscr{L} t}$, for the mean separation (in 3D) between pairs of tracers used in Fig. 13.

measured the average separation of apparent pairs of tracers in simulations by selecting only those initially separated a distance smaller than $2 \mathrm{~mm}$ in the $x-y$ plane, but larger than $1.5 \mathrm{~mm}$ in the $z$ direction. Figure 14 shows the resulting curves, describing a linear growth of the separation, similar to that of the experimental measurements of Figure 12, until the finite size effects of the duct enter into play. The fits of Figure 14 are shown in Table 3, which show a dependence of the rate of separation between tracers with Re similar to the experimental case of Table 1. In this case, an increase by a factor $\simeq 3.3$ of the Reynolds number causes a factor $\simeq 4.4$ in the growth of the separation rate. To allow for a better comparison with experimental results we show in Figure 15 


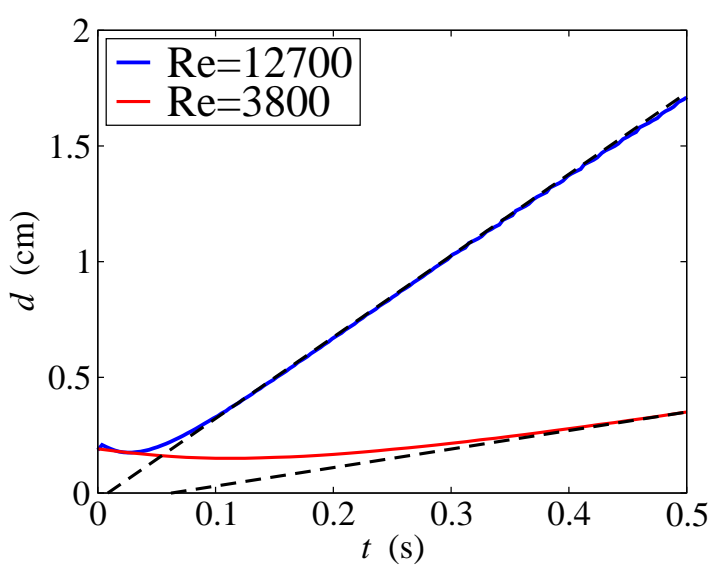

Fig. 15 Mean separation separation $d_{x y}$ (in 2D) between apparent pairs of tracers (i.e., initial $\Delta z_{0}>1.6 \mathrm{~mm}$ ). Fittings in dashed lines, described in Table 3. For $\mathrm{Re}=3800$ the fitting has been calculated in the range $[0.5,3] \mathrm{s}$ (not shown), where the linear behaviour is observed and the fitted line coincides perfectly with the curve.

\begin{tabular}{|c|c||c|c|}
\hline Param & $\operatorname{Re}$ & $d_{0}(\mathrm{~cm})$ & $v_{\text {sep }}(\mathrm{cm} / \mathrm{s})$ \\
\hline \hline$d$ & 3800 & 2.27 & 0.54 \\
$d$ & 12700 & 2.79 & 2.07 \\
\hline \hline$d_{x y}$ & 3800 & -0.05 & 0.80 \\
$d_{x y}$ & 12700 & -0.03 & 3.52 \\
\hline
\end{tabular}

Table 3 Linear fittings of the form $d=d_{0}+v_{\text {sep }} t$, for the mean separation $d$ (in 3D) between apparent pairs of tracers, used in Fig.14. Also mean separation $d_{x y}$ (in 2D) between them to compare with experimental results presented in Table 1. projected 2D distances for apparent tracers. The linear fittings for these results are also included in Table 3.

The last aspect we will analyze concerning the dynamics of bubble pairs is the measurement of the statistics of time needed before a pair separates beyond a minimum distance. In the experimental measurements, as well as in the 376 $_{37}$ simulations, we have considered the time lapse between the moment the pair reduces its separation to a distance smaller ${ }_{378}$ than $2 \mathrm{~mm}$ and the moment it surpasses $4 \mathrm{~mm}$, always taken ${ }_{379}$ between their respective centers. In Figures 16 and $17 \mathrm{we}_{380}$ show the experimental data and the simulated predictions ${ }_{381}$ respectively.

Results are hard to compare due to the large amount ${ }_{303}$ of screening events in the experimental images, that pro- $_{384}$ duce an increasing uncertainty in the shape of the curves as the time lapse grows. In simulations, significant differences are observed between the distribution of probability for real pairs of tracers and that of apparent pairs, with much longe $r_{385}$ life times for real pairs, as a result of the strong correlations of velocities in nearby bubbles, as opposed to the case 386 essentially uncorrelated for distant ones. From the detailed 887 knowledge of the statistics of the time separation of both ap-388 parent and real pairs, taken from numerical simulations to-389 gether with the appropriate characterization of the screening 990 effects, the proper fitting functions could be obtained thator
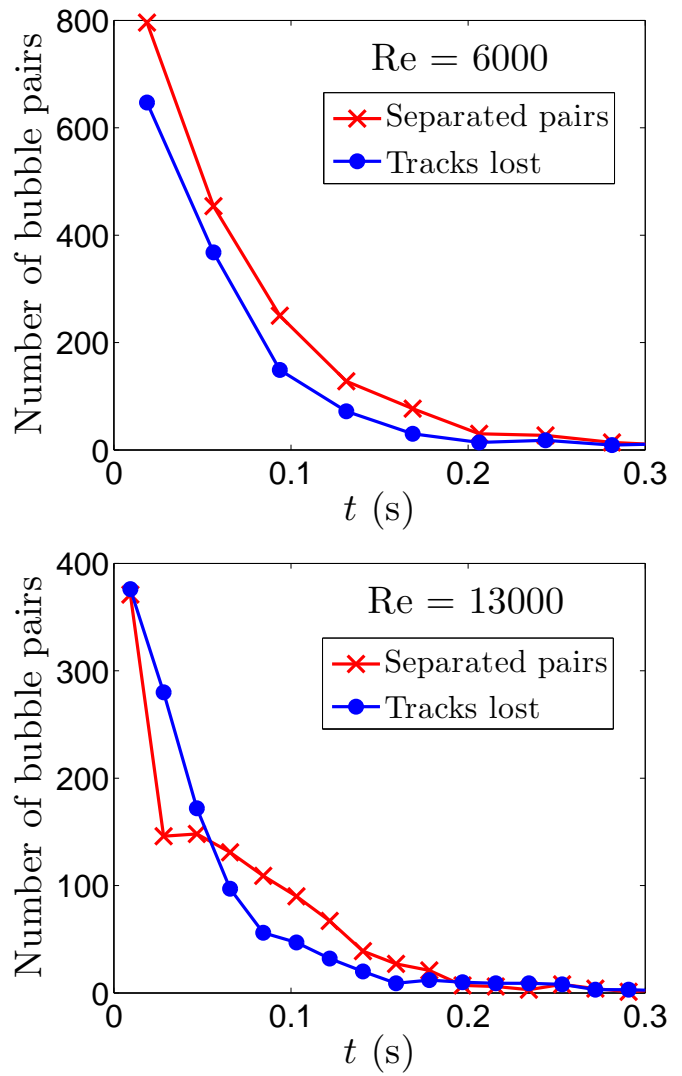

Fig. 16 Time statistics for experimental bubble pairs. Crosses: Distribution of times for the duration of apparent pairs of bubbles (see text); Circles: number of pairs to which we have lost track, during the given time interval, due to screening effects. (Top) Experiment D4, $\mathrm{Re}=6000$. (Bottom) Experiment D3, with $\mathrm{Re}=13000$ (see Bitlloch et al (2018)).

would allow to correctly project the experimental data into a reduced set of parameters in order to extract the statistics of real versus apparent bubble pairs, and thus try to detect whether this observable captures some effect not contained in the passive tracer picture. We have not pursued this idea because, as pointed out before, the limited number of experiments available in microgravity prevents from reaching statistically significant conclusions for the minority of the events of interest, namely those corresponding to the real pairs.

\section{Conclusions}

Large scale Lattice-Boltzmann simulations have been performed to produce reference states of turbulence with the same conditions of the experiments but without bubbles, to contrast with experimental data in the presence of bubbles, in view of detecting nontrivial couplings between bubble dynamics and turbulence. 

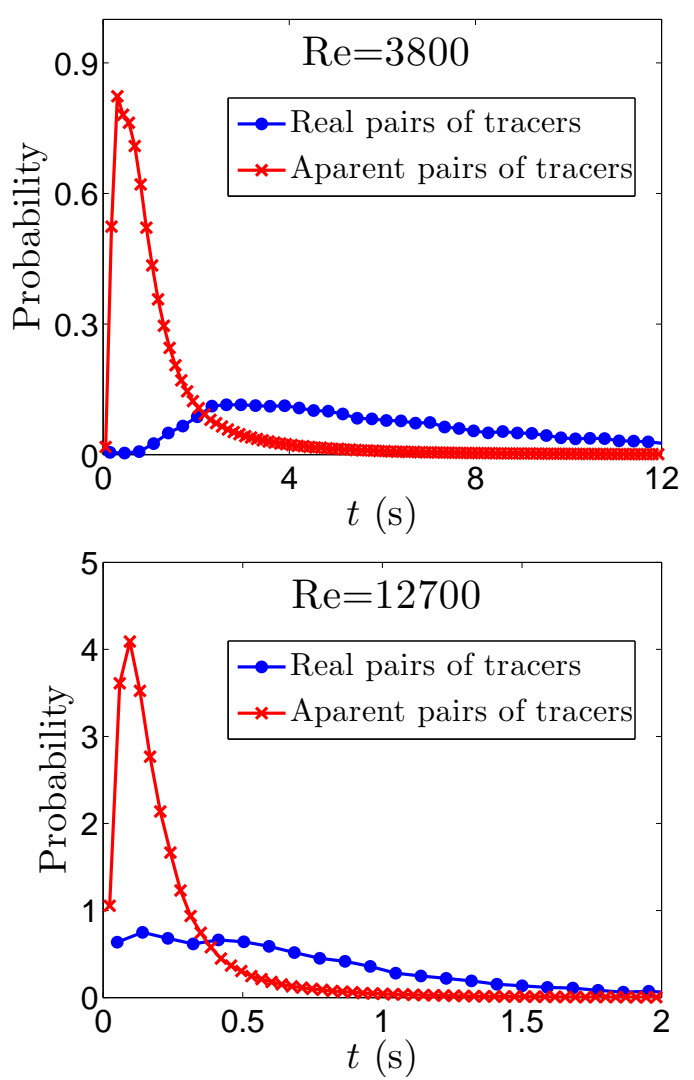

Fig. 17 Normalized probability distribution of duration of pairs of 439 tracers. (Top) $\operatorname{Re}=3800$. (Bottom) $\mathrm{Re}=12700$.
438
This numerical study shows that the relative velocity fluc $_{\bar{\pi}_{43}}$ tuations (scaled to its characteristic velocity) of the flow is s44 $_{44}$ roughly independent of the degree of turbulence, in accor- ${ }_{445}$ dance with the expectation from simple scaling arguments ${ }_{446}$ for fully developed turbulence.

In previous experiments, however, it was observed that 448 the relative velocity fluctuations displayed by bubbles de-449 viated significantly from this scaling, and reflected instead a450 tendency to decrease with increasing Reynolds number. This ${ }_{451}$ suggests an active coupling role of bubbles on the turbulent ${ }_{52}$ flow, that would require a more systematic study to be ${ }^{-{ }^{-5}}{ }^{-453}$ firmed and quantified more precisely.

By using particle tracking we have studied the space-455 time statistics of bubble pairs, and compared it with results\$56 of passive tracers from Lattice-Boltzmann simulations. In ${ }_{457}$ particular we have studied the first-passage time statistics as-458 sociated to the separation of two-close tracers. We find that 59 the average distance between a pair of tracers increases ex-460 ponentially with an effective time scale that depends on the 616 degree of turbulence in the flow. For the case of a pair of ap-462 parent tracers, though, the average separation between thema63 increases linearly with time. In the analysis of experimen-764 tal data, we find a similar behavior for the apparent pairs,465 which dominate the statistics. Real pairs are comparatively $y_{66}$ rare, and any statistical method to extract the corresponding information for those cases would need a larger number of experiments in microgravity. The conclusions of the present analysis could be, in some sense, limited because only 2D projections of the experimental trajectories are available for comparison with numerical results, but demonstrates the use of the recently introduced experimental setup to generate controlled turbulent bubble suspensions in microgravity.

\section{Acknowledgements We acknowledge the support from ESA for the funding of the drop tower experiments that provided the raw data an- alyzed and the ZARM crew, in particular to Dieter Bischoff, for their valuable support all along the experiments and their hospitality. We acknowledges financial support from Ministerio de Economía y Com- petividad (Spain) under projects FIS2013-41144-P, FIS2016-78507- C2-2-P (J.C.), FIS2015-66503-C3-2-P (L.R.-P., also financed by FEDER, European Union), ESP2014-53603-P (X.R.), and Generalitat de Catalunya under projects 2014-SGR-878 (J.C.), 2014-SGR-365 (X.R.). P.B. ac- knowledges Ministerio de Ciencia y Tecnología (Spain) for a pre-doctoral fellowship. We also acknowledge the computing resources, technical expertise and assistance provided by the Barcelona Supercomputing Center, which were financed by RES (Red Española de Supercom- putación, Spain) under projects FI-2010-2-0015, FI-2009-3-0007.}

\section{References}

Arias S, Ruiz X, Casademunt J, Ramírez-Piscina L, González-Cinca R (2009) Experimental study of a microchannel bubble injector for microgravity applications. Microgravity Science and Technology 21(1-2):107-111

Balachandar S, Eaton J (2010) Turbulent dispersed multiphase flow. Annual Review of Fluid Mechanics 42:111133

Bitlloch P (2012) Turbulent bubble suspensions and crystal growth in microgravity. Drop tower experiments and numerical simulations. PhD Thesis

Bitlloch P, Ruiz X, Ramírez-Piscina L, Casademunt J (2015) Turbulent bubble jets in microgravity. Spatial dispersion and velocity fluctuations. Microgravity Science and Technology 27(3):207-220

Bitlloch P, Ruiz X, Ramírez-Piscina L, Casademunt J (2018) Generation and control of monodisperse bubble suspensions in microgravity. Aerospace Science and Technology $77: 344-352$

Carrera J, Ruiz X, Ramírez-Piscina L, Casademunt J, Dreyer M (2008) Generation of a monodisperse microbubble jet in microgravity. AIAA Journal 46(8):2010 - 2019

Colin C (2002) Two-phase bubbly flows in microgravity: some open questions. Microgravity Science and Technology 13(2):16

Colin C, Legendre D, Fabre J (2001) Bubble distribution in a turbulent pipe flow. First International Symposium on Microgravity Research and Applications in Physical Sciences and Biotechnology ESA SP-454 
Colin C, Riou X, Fabre J (2008) Bubble coalescence in gasliquid flow at microgravity conditions. Microgravity Science and Technology 20(3-4):243-246

Hill S, Kostyk C, Motil B, Notardonato W, Rickman S, Swanson T (2010) Thermal management systems roadmap. National Aeronautics and Space Administration

Hou S, Sterling J, Chen S, Doolen GD (1994) A lattice boltzmann subgrid model for high reynolds number flows. Fields Institute Communications 6:1-18

Hurlbert K, Bagdigian B, Carroll C, Jeevarajan A, Kliss M, Singh B (2010) Human health, life support and habitation systems roadmap. National Aeronautics and Space Administration

Kytömaa HK (1987) Stability of the structure in multicomponent flows. Ph.D. Thesis. California Institute of Technology.

Mazzitelli IM, Lohse D, Toschi F (2003) The effect of microbubbles on developed turbulence. Physics of fluids 15(1):L5-L8

Melling A, Whitelaw J (1976) Turbulent flow in a rectangular duct. J Fluid Mech 78(2):289-315

Meyer M, Johnson L, Palaszewsky B, Goebel D, White H, Coote D (2010) In-space propulsion systems roadmap. National Aeronautics and Space Administration

Nourgaliev R, Dinh T, Theofanous T, Joseph D (2003) The lattice boltzmann equation method: theoretical interpretation, numerics and implications. International Journal of Multiphase Flow 29(1):117 - 169

Pattison MJ, Premnath KN, Banerjee S (2009) Computation of turbulent flow and secondary motions in a square duct using a forced generalized lattice boltzmann equation. Phys Rev E 79:026,704

Salazar JP, Collins LR (2009) Two-particle dispersion in isotropic turbulent flows. AnnuRevFluid Mech 41:405432

Tryggvason G, Lu J, Biswas S, Esmaeeli A (2006) Studies of bubbly channel flows by direct numerical simulations. Conference on Turbulence and Interactions TI2006 